\title{
PRIMERAS ETAPAS DEL DESARROLLO \\ DE LA PERSONA: EL DESCUIDO \\ Y LA ÉTICA DEL ESTUPOR
}

Nuevos estilos

$\mathrm{E}$ $\mathrm{n}$ los últimos años, nuevos estilos de vida en cuanto a la concepción y el embarazo se han afirmado. En algunos países europeos, la edad media del primer embarazo aumentó de 25, en 1989, a 27 años, en 1999;' las investigaciones prenatales se han extendido prácticamente a toda la población; las técnicas de fecundación asistida son accesibles a todos.

Frente a estas nuevas tendencias, tha habido una correspondiente mejoría en la salud de los niños? Vatios estudios recientes muestran dudas al respecto.

\section{Edad materna}

Los riesgos inherentes a la edad de la madre son notorios. Recientemente, fue señalado que «el reciente aumento de nacimientos de niños de bajo peso $(<2500 \mathrm{~g})$ y de partos prematuros está en parte ligado al fenómeno de la concepción en edad $>35$ años ; $^{2}$ otros reportan como efectos de la edad 
materna avanzada: mortinatalidad, aborto espontáneo, embarazo ectópico o plurigemelaridad, malformaciones congénitas. Estos datos contrastan con la tendencia de posponer la edad de la primera concepción, se avalan sosteniendo que los nacidos de padres «adultos» tendrían un rendimiento escolar mejor que el de aquellos nacidos de padres jóvenes.

\section{Ecosonografias y amniocéntesis}

En cuanto a las ecografias se debe recordar un estudio detallado y amplio que reporta resultados interesantes: en este estudio, 2.834 mujeres fueron divididas en dos grupos al azat. Un grupo fue sometido a un solo ecosonograma; y otro, a cinco durante el embarazo. La diferencia de crecimiento fetal fue estadísticamente significativa. Esta prueba fue replicada en 1996, pero con resultados menos claros." Recordemos que otro estudio demuestra que dos ccosonogramas durante el embarazo no influyen en la salud del concebido.

Greenough' demostró que los nacidos después de amniocéntesis tienen tiesgo de ingreso en reanimación neonatal, tal vez, por una asociación entre amniocéntesis y problemas respiratotios al nacer; sin embargo, hay que recordar que amniocéntesis y vilocéntesis pueden producir muertes fetales" y anomalías en las piernas. ${ }^{u}$

\section{¿Búsqueda de la perfección o miedo?}

No hay que olvidar que la saña diagnóstica prenatal puede mostrar o provecar problemas de orden psicológico:

Cada exploración fetal, en particular la realización de un cariotipo, provoca, sobre todo en la madre, una verdadera interrupción de la 
relación con el niño, que se reanudará sólo después del resultado de normalidad. A la mínima anomalia la sospecha sobre la calidad del niño induce en los padres una reacción de rechazo totalmente desproporcionada a la gravedad real."

Sabemos que el feto siente este estado de ansia materna, ya que se ha descrito que en caso de depresión $n^{1213} \mathrm{o}$ ansia materna ${ }^{14}$ se van a modificar los parámetros fisiológicos (movimientos, frecuencia cardiaca). Dicha ansia materna se manifiesta como rechazo en el caso de pequeños problemas fetales: (4...] una parte significativa de la población en los países desarrollados y en vias de desarrollo está a favor del aborto selectivo en condiciones como falta de dos dedos, baja estatura, obesidads, " Paradójicamente, algunas veces el hijo es rechazado cuando se prevé que va a nacer indemne de una anomalía, por ejemplo, la sordera, presente en los parientes, ${ }^{16}$ que, a diferencia de lo pensado, quieren un heredero con sus mismos defectos. La Organización Mundial de la Salud invoca una privacidad en lo que respecta al feto para que no se revele, durante los exámenes prenatales de rutina, datos como el sexo o la estatum prevista, que podrian llevar a interrupciones eugenésicas del embarazo."

\section{Fecundaciỏn in vitro}

La fecundación médicamente asistida ha sido ya objeto de señalamientos por su no-inocuidad." Algunos ligan esto no solo a la gemelaridad producida a menudo por esta técnica, sino también a una alteración intrínseca del normal desartollo del cigoto, que al momento de la concepción no tiene contacto con las proteinas producidas por la mucosa tubárica ${ }^{21}$ con posibles efectos aun para los embarazos no gemelares: ${ }^{223}$ "Muchos niños nacidos de una fecundación in vitro - escribe Stromberg en Tbe Lancet- son sanos, pero 
tienen un alto riesgo de discapacidad neurológicas. $Y$ un artículo editorial del mismo número añade; «Los niños nacidos en Suecia con fertilización in vitro tienen tres veces más parálisis cerebral que los concebidos naturalmentes.

En el caso de la técnica ICSI se reportan ulteriores riesgos. ${ }^{2 * 1} \mathrm{La}$ fecundación asistida médicamente es también causa de aumento de ingreso de niños por reanimación neonatal: en Francia esta tasa aumentó de 1995 al 2001 en un $20 \%{ }^{p}$

\section{Iatrogenia}

¿Qué liga a estos cuadros? Contradictoriamente, parece que al crecer las garantias para la madre se reducen las del hijo. Es un fenómena nuevo: la aceptación, tal vez inconsciente, de la alea de producir un daño a la prole.

Hasta ahora se había recurrido a las nuevas técnicas con el objeto de evitar anomalías fetales. Se llega a impedir el nacimiento del fruto de la concepción en un cuadro fundamentalmente centrado en el deseo y el miedo de los padres por su propio smañanas, pero incluyendo también el interés por el mañana del hijo, en una malentendida ecuación entre «normalidad" y ufelicidad». Incluso no se dejaba nacer al hijo aduciendo que uno hubiera sido felizm,

Ahora, el cuadto ha cambiado: el umañana» del hijo se ha vuelto secundario: se cierran los ojos a las consecuencias que los escenarios antes mencionados hacen recaer sobre los hijos y que quedarán indelebles después del nacimiento. «Algunas mujeres quieren un test prenatal precoz independientemente del aumento del riesgo ligado al procedimiento o la relación del riesgo con la posibilidad de una anomalía fetals." Paradójicamente, el 
tien primario a salvaguardar no es ni siquiera el mañana de los padres: las consecuencias de estas actitudes son claramente tales que comprometen un normal desarrollo de la vida social de la pareja. Queda, entonces, como único bien el «hoym, es decir, la satisfacción del narcisismo que no sabe construir, pero que no puede renunciar a la afirmación de sí como ser fecundo. Pero ¿̨fecundo de quién? Fecundo de su proyecto, no más abierto a la gratuidad de un acontecimiento pro creativo que desde siglos lleva aprensión a la familia, pero también estupor.

Así, un escenario nuevo se abre: se considera superado el nivel dèl debate sobre la licitud de la interrupción del embarazo y se pasa a un panorama cuyas posibilidades teratogénicas e iatrogénicas se conocen y se aceptan, con consecuencias patológicas visibles y dolorosas.

Podrímos llamarlo «descuida del Yon, pues lo que constitaye la persona es el deseo, de construir, de vivir, mientras que este escenario nace del miedo de la realidad, del futuro y del deseo mismo.

\section{Ética del estupor}

El miedo al futuro y a lo imprevisto, cha de ser la única ley? Un reciente artículo del Journal of Medical Eishics sugería que cun manantial del renacimiento moral e intelectual para el médico moderno reside en reencontrar el sentido de estupor hacia el cuerpo humano, su puesto en el reino natural, y su milagrosa función como fuente y medio de la experiencia humanas. La palabra "estupon tiene su contrario en la palabra wcontrob. Parece que hoy dia lo ideal es poder tenerlo todo bajo nuestro control. Esto engendra solo estrés. Como uno no puede llegar a conocer, prever, atajarlo todo, siempre habrá algo que sea imprevisto. El imprevisto es el gran enemigo de la sociedad moderna y, 
como la calidad primera que describe el nacimiento es el imprevisto (por lo menos porque no es un automatismo y su fruto se encuentra escondido por nueve meses), este se vive con ansia y miedo. Sin embargo, el imprevisto es la llave de la vida: «Es propio de todo nuevo inicio irrumpir en el mundo como una infinita improbabilidad: pero este "infinitamente improbable" constituye de facto el tejido de todo lo que se llama reabs, ${ }^{\circ}$ y el estupor es la capacidad de reconocer la bondad del imprevisto. Sin estupor el hijo se reduce a (proyecton, a iproducton, y el hombre mismo será ahora proyecto y ahora producto. Sin estupor no hay posibilidad de aliviar el sufrimiento, ya que el estupor (el preguntarse «qquién eres?», «¿̨de quién eres tú, no eres mio?3) posibilita comprender lo que el sufrimiento quiere: empatia y respeto, pero no sentimentalismo: el sentimentalismo es «ser emotivos sin compartins." Qué estupor hay en el reconocer que un niño del tamaño de una mano manifiesta dolor y que en su minúscula manera quiere la salud. Mirar al niño así nos dejó a los clínicos descubrir su dignidad. Descubrimos, así, dos cosas: queel feto (el prematuro) sufrey queestoes aliviado por una presencia.

En dos estudios recientes, " "quisimos estudiar la capacidad de la presencia humana para aliviar el dolor, demostración del profundo deseo de manifestarse presente aun tan precozmente. Estudiamos niños prematuros y a término durante la toma de muestra de sangre usando una técnica de estimulación sensorial para confortar al niño: lo llamamos ssaturación sensoriab. Consiste en distraer y confortar a través del masaje, la palabra, la mirada, ofreciendo manos perfumadas, agua y azúcar en la boca. Registramos una baja en el tiempo de llanto y de tanteo de dolor, respecto de un grupo de control y, también, respecto de grupos de niños a los que, según afirma la literatura, dimos solo agua y azúcar como analgésico. El éxito lo atribuimos a la teoría fisiológica de la cancela de Walls y Melzack," sin embargo, como 
esta teoría comprende dimensiones afectivo-emocionales del dolor, "intuimos que hay algo más, por ello, algunas observaciones ulteriores son necesarias: al dar azúcar dos minutos antes de pinchar el talón o poner un chupón en la boca del recién nacido durante la toma de muestras produce escasa analgesia. $\mathrm{La}$ analgesia es mucho mayot si el recién nacido siente una presencia a su lada. Esto sucede con la técnica que propusimos, pues se establece una relación y los niños no se sienten solos y abandonados durante la experiencia dolorosa. Schopenhauer definió el sufrimiento como la distancia entre lo que demandamos o esperamos de la vida y lo que en realidad obtenemos. ${ }^{*} \mathrm{~L}$ os recién nacidos sienten dolor y tienen deseo: sufren. Eso es porque afirmamos que la intervención médica no debería limitarse a fármacos y procedimientos técnicos. La palabra clave es ipresencian, ya que eso es lo que se requiere y se necesita. Es dificil de suministrar porque no es una medicina y no puede ser dada por un operador desatento; pero es la única manera de reconocer la dignidad del paciente y del operador.

\section{Alexchuir el estupor se engendra dolor}

La verdad es que muy a menudo la respuesta más simple al dolor ha sido su negación. Hasta los años ochenta, tanto por miedo a los efectos colaterales de los analgésicos como por prejuicio, el dolor del recién nacido ha sido siempre negado.

Anand fue el precursor de los estudios sobre el dolor en esta edad de la vida. Demostró en 1985 que el feto y el recién nacido sienten el dolor. Es trastornante pensar que hasta entonces las intervenciones quirúrgicas sobre los recién nacidos se hacian comúnmente sin anestesia." "Como los prematuros y los nacidos a término no tienen memoria del dolor, ellos no 
son probablemente capaces de discriminar el dolor de otros estimulosm. " En 1988, una búsqueda entre los anestesiólogos ingleses e irlandeses mostró que el $60 \%$ de ellos no creía que los recién nacidos sintieran dolor, y solo el $10 \%$ prescribia opióides en el caso de intervenciones quirúrgicas mayores." És clato que con esta actitud se evita considerar al dolor para evitar considerar al paciente y su calidad humana. Es la falta de estupot frente al hombre mismo, frente a la enfermedad,

¿Han cambiado desde entonces las cosas? En teoria si: se han multiplicado los estudios sobre el dolor del recién nacido, y el dolor del feto también ha sido considerado: El feto posee desde el $4 .^{\circ}$ mes de edad gestacional (EG) las estructuras necesarias para la percepción de las diferentes sensaciones: es entonces lógico preocuparse de este dolor posibler;" en otras palabras, seguramente, el feto debe ser salvaguardado del dolor desde las 24 semanas de gestación, pero no podemos excluir que el dolor estuviese presente también antes: «La actitud entre la 15. " y la 24." semana es difícil de codificarse en ausencia de datos precisos sobre el dolor a esa edads." Sin embargo, existen voces discordantes. Un reciente artículo en Pediatric Research titulaba: El feto siente dolor. ¿Esto significa algo para nosotros? $\eta_{;}^{4 a}$ otros articulos escarnecen también el problema: wDolor fetal, un debate infantils, titula Derbyshire en el año 2001. "Todavía hay quien afirma que el feto no puede sentir dolor al no poder decir «Yom; sin embargo, llegan a extender este criterio al recién nacido, así como se niega que el feto sienta el dolor se debe negar que también su análogo extrauterino lo sienta." Como se ve, la idea de sufrimiento es ligada a la de conciencia. Nosotros preferimos atarla a la idea de deseo, según afirma, como vimos, Schopenhauer. Y un feto es todo deseo, ello debido a que es todo potencia, pero también acto. Lo es porque sabe calmarse, sabe obtener, sabe preguntar, claro está, no con las 
palabras. Pero çquién necesita palabras para reconocer la necesidad de un deseo? ¿Quién pretende palabras de un recién nacido, de un minusválido, de un enfermo grave para reconocer que desea estar bien, que desea compañía, que desea salud?

Sin embargo, si se niega que el feto sufra se llega a otras consecuencias. $\mathrm{Y}$ de un error nace el absurdo: hasta se negará que los animales sienten dolor. "Aunque el comportamiento del perro sugiere que tenga dolor, no puedo decir si su relación con el dolor es igual al mío. No sé si el perro está consciente de su cuerpo ni si puedo identificar, reflexionar y controlar su dolon; y se llevan argumentos para identificar la conciencia del perro con la del feto explicando que uno pienso que podemos tener dolor si no somos conscientes de nosotros mismos y de nuestros cuerposy. Pero ¿̨ónde detenerse? Doyal y Wilsher ${ }^{*}$ llegan a afirmar que el niño en los primeros meses de vida no es persona, así como el minusválido («ni los recién nacidos sanos poseen estos atributos, pues no son personas"; kalgunos niños están tan comprometidos mental y fisicamente que su potencial para convertirse en personas está comprometidon). Derbyshire lleva este límite al año de vida atando el estatus de persona a la capacidad de sentir dolor: wa falta de autoconciencia y habilidades conceptuales y simbólicas significa que podemos estar seguros de que toda experiencia fetal y neonatal tiene escasa significación [...], La experiencia del dolor surge aproximadamente a los doce meses de edad $w^{* 6}$ Por su parte, Engehardt aprueba la práctica de someter al recién nacido a procedimientos dolorosos sin anestesia.

Pero partamos de los datos y no de los predatos: el prematuro (cl feto) siente dolor, es capaz de sufrir, y debemos traer a cuento las consecuencias, Nos viene a la memoria el año 1874 , cuando, según algunos relatos, los derechos de los niños se afirmaron por primera vez gracias a la acción de la sociedad 
de los derechos de los animales. ¿Es posible que gatos y ratones tengan garantias en caso de intervención dolorosa y no las renga un feto? Debemos esperar que el feto humano sea equiparado a un animal para que alguien se ocupe de él? ¿Cómo no considerar un derecho el que el feto no reciba dolor?

Sin embargo, frente al dolor de un feto, de un recién nacido, la respuesta no puede ser solo el fármaco. La mirada humana pide dignidad, y este respeto que se le debe implica que no se le infrinja dolor. Por otro lado, el mismo sufrir, o sea, tener un deseo de bienestar, de salud, de bumanidad completa y compartida, afirma la dignidad de quien lo lleva. La persona que sufre no busca solo el fảrmaco, sino una presencia que escuche y acoja su dolor. Para el animal, el problema es la analgesia; para el hombre, el "respeto", es decir, una mirada que no olvide que èl es un valor mayor que la suma de las partes que a una primera mirada parecen componerlo. ¿Cómo no tendirse delante de la presencia de alguien sufriendo, aunque sea escondido y minúsculo, delante de la evidencia de su presencia? ¿Cómo no preguntarse si el dolor es la única via; si la analgesia, la última solución?

Las intervenciones sobre el feto y el recién nacido han de confrontarse con estos dilemas en una sociedad en evolución. El desarrollo de la técrica no puede olvidarse que su objeto es un hombre, y la razón de los investigadores no puede conformarse con una idea de hombre no iluminada por el estupor que permite ver a lo humano en todas (jtodas!) sus formas, kEl hombre no nace para morit, sino para comenzans." 


\section{Notas}

'Serra, G. e I. Bertini. «Nascere nel terzo millenniom. En Il bathbino, suoi diritt, nastri doveri. Siena: Cantagalli, 1999, pp. 17-28.

"Tough, S., C. Newburn-Cook ot al. wDelayed chidbearing and its impact on population rate changes in lower birth weight, multiple birth, and preterm deliverym. Pediatrics, $\mathrm{n},{ }^{\circ} 109$, 2002, pp. 399-403.

'Stein, Z. y M. Susser, «The risks of having children in later lifew. British Medical Journal, $\mathrm{n}$. 320, 2000, pp. 1681-1682.

'Zybert, P., Z. Stein et al. «Maternal age and children's abilitys, Percept Mot Skills, n. ${ }^{\circ} 47,1978$, pp. 815-818.

Newnham, J, S. Evans et al. «Effects of frequentultrasound during pregnancy: a randomised controlled trialy. The Lancet, vol. 342, n. ${ }^{\circ} 8876,1993$, pp. 887-891.

"Evans, S., J. Newnham et al. "Characterisation of the possible effect on birthweight following frequent prenatal ultrasound examinationsm. Early Human Development, vol. $45, \mathrm{n} .^{\circ} 3,19 \mathrm{de}$ julio de 1996,pp. 203-214.

'Koh, S. «The safety of diagnostic continuous wave ultrasonic irradiation-a clinical study serum hemoglobulin level and scanning electron microscopic finding of maternal and cord blood in vitrom. Acta Obstetrita et Gynaecologica Japonica, vol. $33, \mathrm{n}_{+}^{\circ} 4,1981$, pp. 469-478.

'Ewigman, B., J. Crane st ah *Effect of prenatal ultrasound screening on perinatal outcomes, RADIUS Study Group. The Nen England Journal of Medicine, vol. 329, n.' 12, 16 de septiembre de 1993,pp. 821-827.

'Greenough, A., S. Naik ot al, ulnvasive antenatal procedures and requirement for neonatal intensive cate unitadmissiony. EurJ Pediatr, n. ${ }^{\circ} 156,1997$, pp. 550-552.

${ }^{10}$ Alfirevich, Z. «Early amniocentesis versus transabdominal chorion villus sampling for prenatal diagnosism. Cocbrane Database of Systemnatic Reviens, n. ${ }^{\circ}$ 2, 2000. p. CD000077.

"Vial, M., A. Benoit et al, «Maltraitance du foetus et du nouveru-nèv. Ann Pediatr, vol. 43, ni. 6, 1996, pp. 446-455.

"Monk, C. «Stress and mood disorders during ptegnancy: implications for child development\%. Psycbiatr Q, vol.72, n. ${ }^{\circ} 4,2001$, pp. 347-357. 
${ }^{3}$ Allister, L., B. Lestef et al. «The effects of maternal depression on fetal heart rate response to vibroacoustic stimulationv. Developmental Nesropsychology, vol. 20, $\mathrm{n} .{ }^{\circ} 3,2001$, pp. 639-651. ${ }^{\text {"S }}$ jostrom, K, L. Valentin of al. «Maternal anxiety in late pregnancy: effect an fetal movements and fetal heart rates. Early Haman Development, vol. 67, n. ${ }^{\circ} 1-2$, abril de 2002, pp. 87-100.

${ }^{15} \mathrm{Henn}, \mathrm{W}$. «Consumerism in prenatal diagnosis: a challenge for ethical guidelines», Journal of Medical Ethies, vol. 26, n. ${ }^{\circ}$ G, diciembre de 2000, pp. 444-446.

${ }^{16}$ Middileton, A., J. Hewison $t$ al. "Prenatal diagnosis for inherited deafness-what is the potential demand?n. Jourmal of Genetic Coonselling vol. 10, n. ${ }^{\circ} 2$, abril de 2001, pp. 121-131.

${ }^{17}$ World Health Organization: Proposed International guidelines on Etbical Isswes in medical genetics and genetic services. Ginebra: WHO, 1998.

${ }^{18}$ Mitchell, A. unfertility treatment: more risks and challengesw. Tbe New England Josrnal of Medicine, n. ${ }^{\circ} 346,2002$, pp. 769-70.

${ }^{19}$ Koten, G. uAdverse effects of assisted reproductive technology and pregnancy outcome». Pedialric Research, n. ${ }^{\circ} 136,2002$.

${ }^{20}$ Relier, J. unfluence of maternal stress on fetal behavior and brain developmenos. Biology of the Neonate, n. ${ }^{\circ} 79,2001$, pp. 168-71.

${ }^{27}$ Gandolf, F. 3The role of the fallopian tubc. Communication at "The early human life" $n$. Rome, septiembre de 2000,pp. 6-8.

${ }^{2}$ Stromberg, B., G. Dahlquist ef al. «Neurological sequelae in children born after in-vitro fertilisation: a population-based studys. The Lancet, n. ${ }^{\circ} 359,2002, \mathrm{p} .4615$.

${ }^{23}$ Schieve, L., S. Meikle et al. «Low and very low birth weight in infants conceived with use of assisted reproductive technologym. New England Jowmal of Medicine, $n{ }^{\circ} 346,2002, \mathrm{pp}, 731-737$.

${ }^{24}$ Healy, D. y K. Saunders. kFollow-up of children born after in-vitro fertilization\%. The Lancet, n. ${ }^{\circ} 359,2002$.

${ }^{25}$ Hansen, M., J. Kurninczuk et al, «The risk of major birth defects after inmacytoplasmic sperm injection and in vitro fertilizationw. New Englatnd Josirnal of Medicine, n. ${ }^{\circ} 346,2002, \mathrm{pp}$. 725-730.

${ }^{26} \mathrm{Cox}, \mathrm{G}, \mathrm{J}$. Burger of al. \&ntracytoplasmic sperm injection may increase the risk of imprinting defectsm. The American Journal of Human Genetics, vol. 71, n. ${ }^{\circ} 1,2002, \mathrm{pp} .162-164$. ${ }^{27}$ Marnelle, N. uConclusion du rapport AUDIPOG "Santé périnatale 2001" ", Paris, n. ${ }^{*}$, otoño de 2002.

${ }^{21}$ Stranc, L., J. Evans y J. Hamerton. «Chorionic villus sampling and amniocentesis for prenatal diagnosism. The Lancer, n. ${ }^{\circ} 349,1997, \mathrm{pp} .711-714$. 
${ }^{37}$ Evans, M, y D. Greaves. «A renaissance for the sense of wondex? Jonrat of Medital Etbics: Medical Humanities, n. ${ }^{\circ} 27,2001$, p. 1.

${ }^{*}$ Arendt, H. Vita.Arriva. Traducción de G. Zanetri. 5." ed. Milán: Bompiani, 1988, p. 79.

"Scola, A. Salutee Salvexzar wn centro di gratita per la medicina, Siena: Cantagalli, 2000, pp. 30 y 64.

"Bellieni, C., G. Buonocore ef at. aSensorial saturation: an effecrive tool for heel-prick in preterm infantsn. Biology of The Neonate, n. ${ }^{\circ} 80,2001, \mathrm{pp} .15-18$.

"Bellieni, C., F, Bagnoli. NThe effect of multi-sensory stimulation on analgesia in term neonates: a randomized controlled triab). Pediatric Researh, 2002, en imprenca.

"Melzack, R, y P. Wall. wPain mechanisms: s new theoryn. Sience, vol. 150, n. ${ }^{\circ}$ 699, 1965, pp. 971-979.

"Melzack, R. eFrom the gate to the neuromatrixu. Pain Supplement, n. ${ }^{\circ}$ 6, 1999, pp. S121-S126,

${ }^{36}$ Schopenhauer, A. On the basis of morality. Traducción de E. Payne. Indianapolis: BobbsMerrill, 1965, p. 196.

${ }^{5}$ Beyer, J., De Good, D. et al, «Patterns of postoperative anaigesic use with adultes and children following cardiac surgerys. Pain Supplement, vol. 17, n. ${ }^{\circ} 1,1983, \mathrm{pp} .71-81$.

"Richards, T. «Can a fetus feel pain?". Britisb Medical Journal, n. ${ }^{\circ} 291,1985$, pp. 1220-1221.

"De Lima, J., A. Lloyd-Thomas ef al. anfant and neonatal pain: anaesthetists' perceptions and prescribing patternss. Britisb Medical Journal, n. ${ }^{\circ} 313,1996$, p. 787.

"Vial, M. A. Benoit et al. eMaltmitance du foetus et du nouveau-nén. Ann Pédiatr, vol. 43, n. 6, 1996, pp. 446-455.

"Mahicu-Caputo, D, «Prise en charge de la douleur chez le foetus et le nouveau-né. XIVes journées des techniques avancées en gynécologie-obstétrique, PMA et pédiatrie [JTA] „. La Lettre du Gynécologue, n. ${ }^{\circ}$ 242, 1999, pp, 15-21.

"Bhutta, A. «Fetal response to intma-uterine needling: is it pain? Does it matter?3. Pediotric Reseand, 2002.

"Derbyshire, S, «Fetal pain: an infantile debater. Bioethics, vol. 15, n. ${ }^{\circ} 1,2001$, pp. 77-84.

"Englehandt, T. Tbe Foundations of Bioethics. Nueva York: Oxford University Press, 1986, p. 113.

"Doyal, L y D. Wilsher. UTowards guidelines for withholding and withdrawal of life prolonging treatment in neonatal medicinen. Arobives of Disease in Childbeod Fetal Neonatal Ed, n. ${ }^{\circ} 70,1994$, pp. F66-F70.

"Derbyshire, S. docating the beginnings of painm. Bioethes, vol 13, n. ${ }^{\circ} 1,1999$, pp. 1-31.

"Arendt, H., op, it., p. 182 